\title{
Evaluation of Mortality Among Patients of Acute Coronary Syndrome with Frontal QRS-T Angle > 100 Presenting to Tertiary Care Hospital
}

\author{
Haseeb Hassan ${ }^{1}$, Erum Arshad ${ }^{2}$, Muhammad Salman Musharraf ${ }^{3}$, Zaheer Ahmad $^{4}$, Muhammad Zeeshan Aslam ${ }^{5}$ Zakria Anwer $^{6}$
}

1Department of Cardiology, Kingdome of Saudi Arabia, ${ }^{2}$ Department of Medicine, Postgraduate Medical Institute, Lahore-Pakistan, ${ }^{3}$ Department of Cardiology, Faisalabad Institute of
Cardiology, Faisalabad-Pakistan, ${ }^{4}$ Department of Neurology, Allied Hospital, Faisalabad-Pakistan, ${ }^{5}$ Department of Rheumatology, DHQ Hospital, Faisalabad-Pakistan, ${ }^{6}$ Department of
Medicine, Allied Hospital, Faisalabad-Pakistan

\section{ABSTRACT}

Background: Cardiovascular disease (CVD) alone was responsible for $30 \%$ of 58 million deaths world over. ACS includes Unstable angina (UA), nonST elevation myocardial infarction (NSTEMI) and ST elevation myocardial infection (STEMI). Chest pain often described as heaviness, squeezing sensation or discomfort is most commonly reported symptom in patients with ACS. An increasing trend is seen in burden of ACS in Pakistan population with increasing presentation as STEMI. The diagnosis of ACS is usually made on typical changes on electrocardiogram and on raised in cardiac biomarkers. However, studies are being conducted to determine the outcome predictors in patients with ACS. Indeed, relationship exists between PR and QT intervals, heart rate (HR), QRS duration and P, QRS and T waves axis and cardiovascular outcomes. It is of note that the frontal T axis and spatial QRS T angle also predict mortality. Objective: Our objective was to determine the frequency of mortality within 15 days among patients of acute coronary syndrome with frontal QRS-T angle > 100 presenting to tertiary care hospital. Study Design: Descriptive case series. Settings: Department of cardiology, Jinnah hospital, Lahore. Duration: Six months from 19-01-2016 to 19-06-2016. Methodology: One hundred eighty (180) patients presenting to ER department with ACS were offered to be enrolled in the study. Informed consent was obtained from all patients and study protocol was explained. Patients were treated according to department protocols. Patients with QRS-T angle $>100$ was followed for 15 days through regular visits to outdoor departments and telephonic contact. Results: A total of 180 patients presenting to emergency department of cardiology, Jinnah hospital, Lahore with acute coronary syndrome were enrolled in the study. The mean age of the subjects was $52.72 \pm 16.15$ years with minimum and maximum ages of $20 \& 80$ years respectively. In this study $135(75 \%)$ subjects were male while $45(25 \%)$ were females. Frequency of types of Acute Coronary Syndrome was evaluated and $104(57.8 \%)$ cases were found of ST-segment Elevation Ml, 39 (21.7\%) were of Non-ST Elevation MI, while $37(20.6 \%)$ were of unstable Angina. The study results showed that among the subjects, $33(18.3 \%)$ were dead within 15 days, $147(81.7 \%)$ survived among the subjects. So, Mortality rate was found $18.3 \%$ among the subjects. Conclusion: Our study shows that the frequency of mortality seen in patients with QRS - T angles was 18.3\%

Keywords: ACS, QRS - T angle, Cardiac mortality.

Corresponding Author Submitted for Publication: 08-08-2019 Accepted for Publication: 11-08-2020

Dr. Zaheer Ahmad, Senior Registrar, Department of Neurology, Allied Hospital, Faisalabad-Pakistan Email: trueman228@gmail.com

Citation: Hassan H, Arshad E, Musharraf MS, Ahmad Z, Aslam MZ, Anwer Z. Evaluation of Mortality Among Patients of Acute Coronary Syndrome with Frontal QRS-T Angle > 100 Presenting to Tertiary Care Hospital. APMC 2020;14(3):196-9.

\section{DOI: $10.29054 / A P M C / 2020.61$}

\section{INTRODUCTION}

ACS is most common cause of morbidity and mortality across the world. ${ }^{1}$ CVD has been the top most cause of death globally and it is estimated to cause mortality in 17.7 million people representing $31 \%$ of global deaths. ${ }^{2}$

ACS encompasses three conditions: 1) NSTEMI, 2) UA, 3) STEMI. ${ }^{3}$ Central chest pain which is usually heaviness, tightness, squeezing, burning and dull ache is the most common presenting feature of ACS. ACS burden is on up rise in Pakistani population with STEMI as the leading presentation. ${ }^{4}$

ACS diagnosis is usually based on Electrocardiographic changes and raised cardiac biomarkers Trop $T$ and 1.5,6 However, studies are being conducted to determine the outcome predictors in patients with ACS. There has been reported relation between cardiovascular outcomes and ECG changes (PR and QT intervals, heart rate, QRS duration and $P$ - QRS and T - waves axes). ${ }^{7}$ The spatial QRS-T angle and frontal t-axis also predict mortality. ${ }^{8}$ Frontal QRS - T angle can be easily calculated from ECG. There are inconsistent results regarding the mortality in patients with QRS - T angle more than 100. Lown et al showed that the frequency of 2-year mortality seen in patients with ACS has been lowest in patients with frontal QRS - T angles less than $38^{\circ}$ and highest in patients with frontal QRS - T angles more than $104^{\circ}(44.7 \%$ vs $14.8 \%$, p $<0.001){ }^{9}$ Aro et al., that QRS - T angle more than $100^{\circ}$ have been found in $2.0 \%$ of patients, and it is associated with increased risk of sudden cardiac death due to arrhythmia [relative risk (RR) 2.26; 95\% confidence interval (Cl) 1.59-3.21; $P<0.001) .{ }^{10}$ Thus, a wide variation exists between literature with no local study available on this aspect.

This study conducted to determine the frequency of mortality within 15 days among patients of acute coronary syndrome with frontal QRS-T angle > 100 presenting to tertiary care hospital existing literature regarding mortality associates with QRS-T angle $>100$ shows this variation ranging from $17.7 \%$ to $79.9 \%$. Moreover, no local study is available regarding this aspect thus 
this study will bridge this gap and will provide information regarding mortality in patients with QRS-T angle > 100 according to our local population of acute coronary artery syndrome. This will give cardiologist an idea of mortality for better outcome and reduction in mortality and morbidity in these patients. This study will also provide baseline information for further research use of QRS-T angle as outcome predictor and management among patients of coronary artery disease to improve the morbidity and mortality among these patients.

The objective of the study was to determine the frequency of mortality within 15 days among patients of acute coronary syndrome with frontal QRS-T angle > 100 presenting to tertiary care hospital.

\section{METHODOLOGY}

Study Design: Descriptive study.

Settings: Department of cardiology, Jinnah hospital, LahorePakistan.

Duration: Six months from 19-01-2016 to 19-06-2016.

Sample Technique: Patients were enrolled using NonProbability Consecutive sampling.

Sample Size: Estimated sample size was calculated to be 180 at $95 \%$ confidence level. $6 \%$ margin of error and taking mortality about $17.7 \%$ in patients with ACS.

Inclusion Criteria: Both male or female patients of age 20 - 80 years who were new cases of ACS with frontal QRS-T angle more than 100 (as per operational definition) presenting to emergency of tertiary care hospital were enrolled in study while Patients presenting with shock determined by $\mathrm{BP}<90 / 60$ for more than $30 \mathrm{~min}$, patients with history of pervious ischemic heart disease determine on history of myocardial infarction or echocardiography study showing SWMA (segmental wall motion abnormalities like hypo kinetic wall segments), Patients with coronary artery bypass graft determined on history and medical record, patient with history of infective endocarditic determined by medical record and echocardiography showing vegetation, patients with valvular heart disease determined on echocardiography, patients with chronic kidney disease (creatinine clearance $<15 \mathrm{ml} / \mathrm{min}$ ).

Exclusion Criteria: Patients presenting after 24 hours from the beginning of symptoms determined by history and patients dying due to cause other than cardiac determined on history and medical records were excluded from the study.

Methods: ACS was defined as any of the following conditions: ST-Elevation MI (STEMI): Presence of any two of the following will define STEMI.

- $\quad$ ST segment elevation in two consecutive ECG leads (more than $1 \mathrm{~mm}$ in limb leads or more than $2 \mathrm{~mm}$ in chest leads,) with one of the following features

- Raised biomarkers (TROPONIN-T positive)

- Cardiac chest pain (central retrosternal chest pain, persisting for more than 20 minutes)

(b) Non-ST-Elevation MI (NSTEMI): Raised biomarkers with cardiac pain or ST segment depression of more than $0.5 \mathrm{mV}$ or symmetrical $\mathrm{T}$ - waves inversion in two consecutive ECG leads (c) Unstable Angina: Recurrent ischemic chest pains (2 or more episode 10 minutes apart) at rest. Along with ST segment depression more than $0.5 \mathrm{mV}$ in two consecutive ECG leads without raised cardiac biomarkers (TROPONIN T positive)

The frontal QRS - T angle was calculated as the difference between QRS and T-axis. A difference of greater than 100 determine on electrocardiography was labeled as frontal QRS $T$ angle more than 100 . Mortality was measured in terms of death within 15 days from presentation in hospital.

After obtaining informed consent from all patients, study protocol was explained. Patients were treated according to department protocols. Patients with QRS-T angle $>100$ were followed for 15 days through regular visits and data was collected and entered in the SPSS version 20.0. Mean with Standard Deviations were calculated for quantitative variables like age. Frequencies and percentages were calculated in cases of categorical variables like gender, ACS Subtypes and mortality. Data was stratified for age, gender, \& ACS Subtype to see the effect of effect modifiers, Poststratification chi square test was applied. P-value $\leq 0.05$ is considered significant.

\section{RESULTS}

In this present study, a total of 180 patients presenting to emergency department of cardiology, Jinnah hospital, Lahore with ACS were enrolled in the study. The mean age of the subjects was $52.72 \pm 16.15$ years with minimum and maximum ages of $20 \& 80$ years respectively. Table 1

In this study $135(75 \%)$ were male while $45(85 \%)$ were females. Table 1

In our study when frequency of types of acute coronary syndrome was evaluated, $104(57.8 \%)$ cases were found of STElevation Ml, 39 (21.7\%) were of Non-ST Elevation MI, while 37 $(20.6 \%)$ were of unstable angina. Table 1

The study results showed that among the subjects, $33(18.3 \%)$ were dead within 15 days and $147(81.7 \%)$ survived. Mortality rate was found $18.3 \%$ among the subjects. Table 1

Table 1: Descriptive statistics of study population

\begin{tabular}{|l|l|}
\hline \multicolumn{2}{|l|}{ Parameter } \\
\hline Age (Mean + SD) years & $52.72+16.15$ \\
\hline Minimum (years) & 20 \\
\hline Maximum (years) & 80 \\
\hline Gender & $135(75 \%)$ \\
\hline MALE n(5) & $45(25 \%)$ \\
\hline FEMALE & $104(57.5 \%)$ \\
\hline Types of ACS & $39(21.7 \%)$ \\
\hline ST elevation MI & $37(20.6 \%)$ \\
\hline Non-ST elevation MI & $33(18.3 \%)$ \\
\hline Un-Stable Angina & $147(81.7 \%)$ \\
\hline Mortality within 15 days & \\
\hline Yes &
\end{tabular}


Poststratification with respect to age, gender, \& Types of ACS was done to deal with effect modifier. Table 2 - 5 .

Table 2: Mortality among the subtypes of acute coronary syndrome subtypes

\begin{tabular}{|c|c|c|c|c|}
\hline \multirow{2}{*}{ ACS } & \multicolumn{3}{|c|}{$\begin{array}{c}\text { Mortality Within 15 } \\
\text { Days }\end{array}$} & \multirow{2}{*}{$\begin{array}{c}\text { T- } \\
\text { value }\end{array}$} \\
\cline { 2 - 4 } & Yes & No & & \\
\hline St-Elevation MI & 19 & 85 & $\mathbf{1 0 4}$ & \\
\hline Non St-Elevation MI & 8 & 31 & 39 & \multirow{2}{*}{$\mathbf{0 . 8 9}$} \\
\hline Unstable Angina & 6 & 31 & $\mathbf{3 7}$ & \\
\hline Total & $\mathbf{3 3}$ & $\mathbf{1 4 7}$ & $\mathbf{1 8 0}$ & \\
\hline
\end{tabular}

Table 3: Stratification of Mortality among the subtypes of Acute Coronary Syndrome subtypes w.r.t Age categories

\begin{tabular}{|c|c|c|c|c|}
\hline \multirow{2}{*}{ Age Categories } & \multicolumn{2}{|c|}{ Mortality Within 15 Days } & \multirow{2}{*}{ Total } & p-value \\
\cline { 2 - 4 } & Yes & No & & \\
\hline $20-40$ yrs. & 7 & 37 & 44 & \\
\hline $41-60$ yrs. & 12 & 56 & 68 & \multirow{2}{*}{0.031} \\
\hline $61-80$ yrs. & 14 & 54 & 68 & \\
\hline Total & 33 & 147 & 180 & \\
\hline
\end{tabular}

Table 4: Stratification of Mortality among the subtypes of Gender category

\begin{tabular}{|c|c|c|c|c|}
\hline \multirow{2}{*}{ Gender } & \multicolumn{2}{|c|}{ Mortality Within 15 Days } & \multirow{2}{*}{ Total } & \multirow{2}{*}{ p-value } \\
\cline { 2 - 4 } & Yes & No & & \\
\hline Male & 25 & 110 & 135 & \multirow{2}{*}{0.010} \\
\hline Female & 8 & 37 & 45 & \\
\hline Total & 33 & 147 & 180 & \\
\hline
\end{tabular}

Table 5: Stratification of Mortality among the subtypes of ACS subtypes

\begin{tabular}{|c|c|c|c|c|}
\hline \multirow{2}{*}{ ACS } & \multicolumn{2}{|c|}{$\begin{array}{c}\text { Mortality Within 15 } \\
\text { Days }\end{array}$} & \multirow{2}{*}{ Total } & $\begin{array}{c}\text { p- } \\
\text { value }\end{array}$ \\
\cline { 2 - 4 } & Yes & No & & \\
\hline St-Elevation MI & 19 & 85 & 104 & \\
\hline Non St-Elevation MI & 8 & 31 & 39 & \multirow{2}{*}{0.023} \\
\hline Unstable Angina & 6 & 31 & 37 & \\
\hline Total & 33 & 147 & 180 & \\
\hline
\end{tabular}

\section{DISCUSSION}

ACS is a very common cause of morbidity and mortality across the world. CVD alone was responsible for $31 \%$ of Global death toll.

The ECG is the most readily accessible and commonly used diagnostic investigation to guide emerging cardiac catastrophes like ACS. The ECG done during an ACS serves as an invaluable investigation of diagnostic, prognostic and therapeutic significance. ${ }^{11}$ Previous studies have shown that ECG findings like heart rate, left ventricular mass (voltage, QRS axis),
conduction(Pwave duration, PR interval, QRS duration) and repolarization (ST segment deviation, ST segment slope, QT interval, T wave amplitude) are of prognostic value, patients with hypertension, heart failure, resuscitated patients after cardiac arrest, coronary artery bypass grafting, and in patients in clinical trials. ${ }^{12-18}$

There are multiple tools available to assist in cardiac mortality risk stratification and ECG happens to be available most commonly and cheaply in our setup. It is noninvasive, inexpensive, less human resource utilization and gives quick results. However, it is underutilized in stratification of cardiac mortality risk mainly because of unawareness among medical professionals. During last decade, there has been several studies reported to predict mortality in cardiovascular diseases on electrocardiographic changes.

Our study was a small-scale study with only 180 enrolled patients. In this study, frequency of types of ACS was found to be $104(57.8 \%)$ cases of STEMI, $39(21.7 \%)$ of NSTEMI, while $37(20.6 \%)$ were of UA. The study results showed that among the subjects, $33(18.3 \%)$ were dead within 15 days and 147 $(81.7 \%)$ survived. So, Mortality rate was found to be $18.3 \%$.

Though very little data is available on the subject, however few studies that we found showed that there is a great inconsistency and variation among the studies. Lown et al showed a 2-year mortality of $44.7 \%$ in subjects with ACS having QRS-T angle > 100 however, no short-term mortality data is available which can be compared to our study. ${ }^{9}$

\section{CONCLUSION}

It was concluded that the frequency of mortality seen in patients with QRS - T angles >100 was $18.3 \%$ in Pakistani population.

\section{LIMITATIONS}

No other study on mortality among patients of ACS with frontal QRS-T angle $>100$ was found on literature search.

\section{SUGGESTIONS / RECOMMENDATIONS}

It is recommended that further large-scale studies should be conducted on this topic which will help us ascertain the prognostic value of this invaluable tool in predicting cardiovascular mortality in ACS.

\section{CONFLICT OF INTEREST / DISCLOSURE None.}

\section{ACKNOWLEDGEMENTS}

We are thankful to our colleagues and patients who contributed in this study.

\section{REFERENCES}

1. Switaj TL, Christensen SR, Brewer DM. Acute Cornoary syndrome: Current Treatment. Am Fam Physician. 2017;95(4):232-40.

2. World Health Organization. (2018). Cardiovascular diseases (CVDs). [online] Available at: http://www.who.int/news-room/fact- 
sheets/detail/cardiovascular-diseases-(cvds) [Accessed 7 Aug. 2018].

3. Kumar, A. and Cannon, C. Acute Coronary Syndromes: Diagnosis and Management, Part I. Mayo Clinic Proceedings. 2009;84(10):10:917-38.

4. Noor L, Adnan Y,Khan SB, Rehman H, Ahmad F, Hafizullah M. Changing trend of presentation of acute coronary syndrome in peshawar over the last sixteen years. J Ayub Med Coll Abbottabad. 2011;23(2):136-9.

5. Chacko S, Haseeb S, Glover BM, Wallbridge D, Harper A. The role of biomarkers in the diagnosis and risk stratification of acute coronary syndrome. Future Science OA. 2018;4(1):FSO251.

6. Nikus K, Birnbaum Y, Eskola M, Sclarovsky S, Zhong-qun Z, Pahlm O. The role of biomarkers in the diagnosis and risk stratification of acute coronary syndrome. Future Science OA. 2018;4(1):FSO251.

7. Mozos, I. and Caraba, A. Electrocardiographic Predictors of Cardiovascular Mortality. Dis Markers. 2015;2015:727401.

8. Zhang, X., Zhu, Q., Zhu, L., Jiang, H., Xie, J., Huang, W. and Xu, B. (2015). Spatial/Frontal QRS-T Angle Predicts All-Cause Mortality and Cardiac Mortality: A Meta-Analysis. PLoS One. 2015;10(8):e0136174.

9. Lown M, Munyombwe T, Harrison W, West R, Hall C, Morrell C, et al. Association of Frontal QRS-T Angle-Age Risk Score on Admission Electrocardiogram with Mortality in Patients Admitted with an Acute Coronary Syndrome $\dagger$. Am J Cardiolo. 2012;109(3):307-13.

10. Aro A, Huikuri $H$, Tikanen J, Junttila $M$, Rissanen $H$, Reunanen A. Anttonen, O. QRS-T angle as a predictor of sudden cardiac death in a middle-aged general population. Europace. 2010;14(6):872-6.

11. Nikus, K, Pahlm, O, Wagner G, Birnbaum Y, Cinca J, Clemmensen P, Eskola M. Electrocardiographic classification of acute coronary syndromes: a review by a committee of the International Society for Holter and Non-Invasive Electrocardiology. J Electrocardiol. 2010;43(2):91-103.

12. Levy D, Salomon M, D'Agostino RB, et al. Prognostic implications of baseline electrocardiographic features and their serial changes in subjects with left ventricular hypertrophy. Circulation. 1994;90(4):1786-93.

13. Okin PM, Devereux RB, Kors JA. Computerized ST depression analysis improves prediction of all-cause and cardiovascular mortality: the strong heart study. Ann Noninvasive Electrocardiol. 2001;6(2):107-16.

14. Okin PM, Devereux RB, Nieminen MS. Electrocardiographic strain pattern and prediction of cardiovascular morbidity and mortality in hypertensive patients. Hypertension. 2004;44(1):4854.

15. Haissaguerre M, Derval N, Sacher F. Sudden cardiac arrest associated with early repolarization. $\mathrm{N}$ Engl $\mathrm{J}$ Med. 2008;358(19):2016-23.

16. Lauer MS, Martino D, Ishwaran H. Quantitative measures of electrocardiographic left ventricular mass, conduction, and repolarization, and long-term survival after coronary artery bypass grafting. Circulation. 2007;116(8):888-93.

17. Denes P, Larson JC, Lloyd-Jones DM Major and minor ECG abnormalities in asymptomatic women and risk of cardiovascular events and mortality. JAMA. 2007;297(9):978-85.

18. Prineas RJ, Grandits G, Rautaharju PM. Long-term prognostic significance of isolated minor electrocardiographic T-wave abnormalities in middle-aged men free of clinical cardiovascular disease (The Multiple Risk Factor Intervention Trial [MRFIT]). Am J Cardiol. 2002;90(12):1391-5.

\section{AUTHORSHIP CONTRIBUTION}

\begin{tabular}{|l|l|}
\hline Dr. Haseeb Hassan & \\
Consultant Cardiologist, Kingdome of & Principal Investigator \\
Saudi Arabia, & \\
\hline $\begin{array}{l}\text { Dr. Erum Arshad } \\
\text { Post Graduate Trainee, Department of } \\
\text { Medicine, Postgraduate Medical } \\
\text { Institute, Lahore-Pakistan }\end{array}$ & Co-Investigator \\
\hline $\begin{array}{l}\text { Dr. Muhammad Salman Musharraf } \\
\text { Medical Officer, Department of } \\
\text { Cardiology, Faisalabad Institute of } \\
\text { Cardiology, Faisalabad-Pakistan }\end{array}$ & \\
\hline $\begin{array}{l}\text { Dr. Zaheer Ahmad } \\
\text { Senior Registrar, Department of }\end{array}$ & \\
Neurology, Allied Hospital, Faisalabad- & \\
Pakistan & Statistical Analysis \\
\hline Dr. Muhammad Zeeshan Aslam & \\
Consultant Rheumatologist, DHQ & Co-Investigator \\
Hospital, Faisalabad-Pakistan & \\
\hline Dr. Zakria Anwer & \\
Senior Registrar, Medicine & References Layout \\
Allied Hospital, Faisalabad Pakistan & \\
\hline
\end{tabular}

